CERN-PH-TH/2005-014

DCPT-04-114

IPPP-04-57

TSL/ISV-2005-0287

UWThPh-2004-28

WUE-ITP-2005-001

hep-ph/0502036

\title{
Distinguishing between MSSM and NMSSM by combined LHC and ILC analyses
}

\author{
G. Moortgat-Pick ${ }^{a}$, S. Hesselbach ${ }^{b, c}$, F. Franke $^{d}$, H. Fraas $^{d}$ \\ ${ }^{a}$ TH Division, Physics Department, CERN, CH-1211 Geneva 23, Switzerland \\ ${ }^{b}$ Institut für Theoretische Physik, Universität Wien, A-1090 Vienna, Austria \\ ${ }^{c}$ High Energy Physics, Uppsala University, Box 535, S-751 21 Uppsala, Sweden \\ ${ }^{d}$ Institut für Theoretische Physik und Astrophysik, Universität Würzburg, \\ Am Hubland, D-97074 Würzburg, Germany
}

\begin{abstract}
We show that the interplay between the LHC and the $e^{+} e^{-}$International Linear Collider (ILC) with $\sqrt{s}=500 \mathrm{GeV}$ might be crucial for the discrimination between the minimal and next-to-minimal supersymmetric standard model. We present an NMSSM scenario that cannot be distinguished from the MSSM by cross sections and mass measurements if only the light neutralinos and the lightest chargino are kinematically accessible, even if one of the neutralinos has a significant singlino component. Mass predictions for the heavier neutralinos from the ILC analysis and their observation at the LHC lead to an identification of the neutralino mixing character and the underlying supersymmetric model in a combined LHC/ILC analysis. In our numerical example we include errors in the mass measurements and use standard methods of supersymmetric parameter determination.
\end{abstract}




\section{Introduction}

The International Linear Collider (ILC) is intended to start with an energy of $\sqrt{s}=$ $500 \mathrm{GeV}$, which will be upgraded to about $1 \mathrm{TeV}$ [1]. The physics program includes the complement of the results at the Large Hadron Collider (LHC) [2] and the discovery of possible signatures of physics beyond the standard model (SM). Since supersymmetry is often considered as the main candidate for new physics, the precise resolution of the supersymmetric model is one of the essential goals of the ILC [3].

An interesting possibility for the determination of the supersymmetric model is to study the gaugino/higgsino particles, which are expected to be among the lightest supersymmetric particles. In this paper we consider two basic supersymmetric models: the minimal supersymmetric standard model (MSSM) and the next-to-minimal supersymmetric standard model (NMSSM). The MSSM contains four neutralinos $\tilde{\chi}_{i}^{0}$, which are mixtures of the photino, zino and neutral higgsinos, and two charginos $\tilde{\chi}_{i}^{ \pm}$, being mixtures of wino and charged higgsino. The neutralino/chargino sector depends at tree level on four parameters: the $\mathrm{U}(1)$ and $\mathrm{SU}(2)$ gaugino masses $M_{1}$ and $M_{2}$, the higgsino mass parameter $\mu$, and the ratio $\tan \beta$ of the vacuum expectation values of the Higgs fields. For the determination of these parameters, straightforward strategies [4, 5] have been worked out even if only the light neutralinos and charginos $\tilde{\chi}_{1}^{0}, \tilde{\chi}_{2}^{0}$ and $\tilde{\chi}_{1}^{ \pm}$are kinematically accessible at the first stage of the ILC [6].

The NMSSM [7] is the simplest extension of the MSSM by an additional Higgs singlet field. New parameters in the neutralino sector are the vacuum expectation value $x$ of the singlet field and the trilinear couplings $\lambda$ and $\kappa$ in the superpotential, where the product $\lambda x=\mu_{\text {eff }}$ replaces the $\mu$-parameter of the MSSM [8, 9]. The additional fifth neutralino may significantly change the phenomenology of the neutralino sector. In scenarios where the lightest supersymmetric particle is a nearly pure singlino, the existence of displaced vertices leads to a particularly interesting experimental signature [10, 11]. If the complete neutralino sector is accessible, the sum rules for the production cross sections show a different energy dependence in MSSM and NMSSM [6]. The NMSSM Higgs sector also depends on the soft scalar mass parameters $A_{\lambda}$ and $A_{\kappa}$. It contains five physical neutral Higgs bosons, three scalars $S_{i}$ and two pseudoscalars $P_{i}$. In the following we will assume that only the light neutralinos, charginos and the lightest Higgs boson $S_{1}$ are accessible at the first stage of the linear collider.

It has already been worked out that there exist MSSM and NMSSM scenarios with the same mass spectra of the light neutralinos but different neutralino mixing. In this case beam polarization is crucial for distinguishing the two models [12]. We present a scenario where all kinematically accessible neutralinos and charginos have similar masses and almost identical cross sections, within experimental errors, in MSSM and NMSSM. Although the second lightest neutralino in the NMSSM has a significant singlino component, the models cannot be distinguished by precision measurements of masses and cross sections at the LHC or at the ILC with $\sqrt{s}=500 \mathrm{GeV}$. While the branching ratios for the neutralino decays into Higgs bosons may give first evidence, final identification of the underlying model requires precision measurements of the masses and mixing characters of the heavier neutralinos by combined analyses of LHC and $\operatorname{ILC}_{500}$ results and by exploiting the $\operatorname{ILC}_{650}^{\mathcal{L}=1 / 3}$ option with $\sqrt{s}=650 \mathrm{GeV}$ at the cost 
of reduced luminosity. The $\mathrm{ILC}_{650}$ option reflects the high flexibility of the ILC and does not require any specific setups in the experiment. Of course, the second energy stage of the ILC with energies up to $1 \mathrm{TeV}$ also allows a discrimination. Here, however, we focus on the potential of the ILC $_{500 / 650}$.

This paper is organized as follows: in Section 2 we describe in detail our strategy for the discrimination between NMSSM and MSSM. Our analysis is based on the scenario presented in Section 3. The following sections 4, 5 and 6 contain the numerical results for the parameter determination at $\mathrm{ILC}_{500}, \mathrm{LHC}$ and $\mathrm{ILC}_{650}$. We estimate errors in the mass measurements and include statistical errors for the cross sections. Fit programs, which exist so far only for the MSSM [13], are not used. Special attention is given to the interplay in combined analyses of the experimental results at ILC and LHC.

\section{Strategy}

We consider an NMSSM scenario where in the gaugino/higgsino sector only the masses of the light chargino $\tilde{\chi}_{1}^{ \pm}$and the light neutralinos $\tilde{\chi}_{1}^{0}$ and $\tilde{\chi}_{2}^{0}$ are accessible at the ILC $_{500}$. We calculate the masses of the charginos and neutralinos and the cross sections for the pair production of the light chargino $e^{+} e^{-} \rightarrow \tilde{\chi}_{1}^{+} \tilde{\chi}_{1}^{-}$and for the associated production of the light neutralinos $e^{+} e^{-} \rightarrow \tilde{\chi}_{1}^{0} \tilde{\chi}_{2}^{0}$ with polarized and unpolarized beams.

The masses and cross sections provide the experimental input for deriving the supersymmetric parameters within the MSSM using standard methods [5, 6]:

- We assume an uncertainty of $1-2 \%$ for the masses $m_{\tilde{\chi}_{1}^{ \pm}}, m_{\tilde{\chi}_{1}^{0}}, m_{\tilde{\chi}_{2}^{0}}, m_{\tilde{\nu}_{e}}, m_{\tilde{e}_{L}}$ and $m_{\tilde{e}_{R}}$. In the cross sections for $e^{+} e^{-} \rightarrow \tilde{\chi}_{1}^{+} \tilde{\chi}_{1}^{-}$and $e^{+} e^{-} \rightarrow \tilde{\chi}_{1}^{0} \tilde{\chi}_{2}^{0}$ we include a statistical error of one standard deviation and take into account the error due to the polarization uncertainty of $\Delta \mathcal{P}_{e^{ \pm}} / \mathcal{P}_{e^{ \pm}}=0.5 \%$.

- From the chargino mass $m_{\tilde{\chi}_{1}^{ \pm}}$and the cross section $e^{+} e^{-} \rightarrow \tilde{\chi}_{1}^{+} \tilde{\chi}_{1}^{-}$measured at two energies, $\sqrt{s}=400 \mathrm{GeV}$ and $500 \mathrm{GeV}$, we determine bounds for the elements $U_{11}$ and $V_{11}$ of the chargino mixing matrices. Polarized beams allow the resolution of ambiguities and the improvement of the accuracy.

- Using the mixing matrix elements $U_{11}$ and $V_{11}$, the masses $m_{\tilde{\chi}_{1}^{ \pm}}, m_{\tilde{\chi}_{1}^{0}}$ and $m_{\tilde{\chi}_{2}^{0}}$, and the cross sections for $e^{+} e^{-} \rightarrow \tilde{\chi}_{1}^{0} \tilde{\chi}_{2}^{0}$, we derive constraints for the parameters $M_{1}$, $M_{2}, \mu$ and $\tan \beta$.

- After the determination of the fundamental MSSM parameters we calculate the heavy chargino and neutralino masses and compare them with LHC analyses [14].

The experimental results from the ILC with $\sqrt{s}=400 \mathrm{GeV}$ and $500 \mathrm{GeV}$ lead to consistent MSSM parameters despite the fact that we start with an NMSSM scenario where the light neutralino $\tilde{\chi}_{2}^{0}$ has a significant singlino component. In the considered scenario the ILC $_{500}$ does not allow a discrimination between MSSM and NMSSM by mass and cross section measurements. Then the identification of the underlying supersymmetric 
model is performed in combined analyses at the $\mathrm{LHC}$ or the $\operatorname{ILC}_{650}^{\mathcal{L}=1 / 3}$ by the measurement of the masses of the heavy neutralinos and charginos.

\section{Scenario}

We discuss an NMSSM scenario where the lightest visible neutralino $\tilde{\chi}_{2}^{0}$ is accessible at the $\mathrm{ILC}_{500}$ and has a significant singlino component. The supersymmetric parameters and the resulting masses and neutralino mixing states are given in Table 1. For the basis in the neutralino system we use the conventions of [15, 16]. The hierarchy $M_{1}>M_{2}$ of the $\mathrm{U}(1)$ and $\mathrm{SU}(2)$ mass parameters causes an approximate mass degeneration of the lightest neutralino $\tilde{\chi}_{1}^{0}$, which is assumed to be the lightest supersymmetric particle (LSP), and the light chargino $\tilde{\chi}_{1}^{ \pm}$that is typical for a minimal anomaly mediated SUSY-breaking mechanism (mAMSB) [17]. Even small mass differences may be resolved experimentally with good accuracy at the LHC [18] and by applying the ISR method [19] at the linear collider [20]. One should note, however, that we do not use any assumptions on possible SUSY-breaking mechanisms for the following parameter determination. For comparison Table 1 also contains the parameters of the MSSM scenario derived in Section 4, which leads to indistinguishable mass spectra and cross sections within the experimental errors at the $\mathrm{ILC}_{500}$. All parameters are in agreement with bounds from dark matter constraints [21].

Table 2 shows the masses and mixings of the NMSSM Higgs bosons for parameters $A_{\lambda}$ and $A_{\kappa}$ within the theoretical and experimental constraints in the NMSSM scenario

$$
\begin{aligned}
2740 \mathrm{GeV} & <A_{\lambda}<5465 \mathrm{GeV}, \\
-553 \mathrm{GeV} & <A_{\kappa}<0 \mathrm{GeV} .
\end{aligned}
$$

Here the light scalar $S_{1}$ has MSSM-like character while the second lightest scalar $S_{2}$ and the light pseudoscalar $P_{1}$ are almost pure singlets with masses larger than $200 \mathrm{GeV}$ for $-443 \mathrm{GeV}<A_{\kappa}<-91 \mathrm{GeV}$. Within this $A_{\kappa}$ region direct Higgs production does not allow the identification of the NMSSM [22] since scalar and pseudoscalar Higgs bosons with dominant singlet character escape detection. Detailed studies on the detection of light singlet dominated Higgs bosons at the LHC and ILC can be found in [23], and of light pseudoscalars in scenarios with dominant decay $S_{1} \rightarrow P_{1} P_{1}$ at LHC, ILC and the photon collider in [24].

Due to the mass difference between the light neutralinos in our scenario the decays of $\tilde{\chi}_{2}^{0}$ and $\tilde{\chi}_{3}^{0}$ into $S_{2}$ and $P_{1}$ are kinematically forbidden. The branching ratio for the decay of $\tilde{\chi}_{2}^{0}$ into the lightest Higgs boson $S_{1}$ is enhanced by a factor two to about $20 \%$ in the NMSSM scenario compared to the MSSM. Therefore precise measurements of the neutralino decays into Higgs bosons could provide first evidence for the underlying model that has to be confirmed by the combined LHC and ILC analyses described in the next sections. 


\begin{tabular}{|l|c|c|}
\hline & NMSSM & MSSM \\
\hline \hline$M_{1}$ & $360 \mathrm{GeV}$ & $375 \mathrm{GeV}$ \\
$M_{2}$ & $147 \mathrm{GeV}$ & $152 \mathrm{GeV}$ \\
$\tan \beta$ & 10 & 8 \\
$\mu$ & - & $360 \mathrm{GeV}$ \\
$\lambda$ & 0.5 & - \\
$x$ & $915 \mathrm{GeV}$ & - \\
$\kappa$ & 0.2 & - \\
\hline \hline$m_{\tilde{\chi}_{1}^{0}}$ & $138 \mathrm{GeV}$ & $138 \mathrm{GeV}$ \\
$m_{\tilde{\chi}_{2}^{0}}$ & $337 \mathrm{GeV}$ & $344 \mathrm{GeV}$ \\
$m_{\tilde{\chi}_{3}^{0}}$ & $367 \mathrm{GeV}$ & $366 \mathrm{GeV}$ \\
$m_{\tilde{\chi}_{4}^{0}}$ & $468 \mathrm{GeV}$ & $410 \mathrm{GeV}$ \\
$m_{\tilde{\chi}_{5}^{0}}$ & $499 \mathrm{GeV}$ & - \\
\hline$m_{\tilde{\chi}_{1}^{ \pm}}$ & $139 \mathrm{GeV}$ & $139 \mathrm{GeV}$ \\
$m_{\tilde{\chi}_{2}^{ \pm}}$ & $474 \mathrm{GeV}$ & $383 \mathrm{GeV}$ \\
\hline$m_{\tilde{e}_{L}}$ & $240 \mathrm{GeV}$ & $240 \mathrm{GeV}$ \\
$m_{\tilde{e}_{R}}$ & $220 \mathrm{GeV}$ & $220 \mathrm{GeV}$ \\
$m_{\tilde{\nu}_{e}}$ & $226 \mathrm{GeV}$ & $226 \mathrm{GeV}$ \\
\hline \hline$\tilde{\chi}_{1}^{0}$ mixing state & $(-0.02,0.97,-0.20,0.09,-0.07)$ & $(0.03,-0.96,0.26,-0.13)$ \\
$\tilde{\chi}_{2}^{0}$ mixing state & $(0.62,0.14,0.25,-0.31,0.65)$ & $(0.72,0.22,0.48,-0.46)$ \\
$\tilde{\chi}_{3}^{0}$ mixing state & $(-0.75,0.04,0.006,-0.12,0.65)$ & $(-0.04,0.10,-0.70,-0.71)$ \\
$\tilde{\chi}_{4}^{0}$ mixing state & $(-0.03,0.08,0.70,0.70,0.08)$ & $(-0.70,0.18,0.47,-0.52)$ \\
$\tilde{\chi}_{5}^{0}$ mixing state & $(0.21,-0.16,-0.64,0.62,0.37)$ & - \\
\hline
\end{tabular}

Table 1: Parameters, mass eigenvalues and neutralino mixing states in the basis $\left(\tilde{B}^{0}, \tilde{W}^{0}, \tilde{H}_{1}^{0}, \tilde{H}_{2}^{0}, \tilde{S}\right)$ in the NMSSM scenario. For comparison the table also contains an MSSM scenario (neutralino basis $\left.\left(\tilde{B}^{0}, \tilde{W}^{0}, \tilde{H}_{1}^{0}, \tilde{H}_{2}^{0}\right)\right)$ with a similar mass spectrum of the light neutralinos and charginos but different neutralino mixing. 


\begin{tabular}{|l|c|}
\hline$A_{\lambda}$ & $4000 \mathrm{GeV}$ \\
$A_{\kappa}$ & $-200 \mathrm{GeV}$ \\
\hline$m_{S_{1}}$ & $124 \mathrm{GeV}$ \\
$m_{S_{2}}$ & $311 \mathrm{GeV}$ \\
$m_{S_{3}}$ & $4347 \mathrm{GeV}$ \\
$m_{P_{1}}$ & $335 \mathrm{GeV}$ \\
$m_{P_{2}}$ & $4347 \mathrm{GeV}$ \\
$m_{H^{ \pm}}$ & $4346 \mathrm{GeV}$ \\
\hline$S_{1}$ mixing state & $(-0.9947,-0.0990,0.0292)$ \\
$S_{2}$ mixing state & $(-0.0271,-0.0222,-0.9994)$ \\
$S_{3}$ mixing state & $(0.0996,-0.9948,0.0193)$ \\
$P_{1}$ mixing state & $(0.0016,0.0164,-0.9999)$ \\
$P_{2}$ mixing state & $(0.0995,0.9949,0.0165)$ \\
\hline
\end{tabular}

Table 2: The parameters $A_{\lambda}$ and $A_{\kappa}$ of the NMSSM Higgs sector and the resulting masses and mixing states of the Higgs particles according to NMHDECAY [15] for the parameters in the neutralino sector as given in Table 1 and $M_{Q}=M_{U}=M_{D}=800 \mathrm{GeV}$ and $A_{t}=A_{b}=1500 \mathrm{GeV}$.

\section{Parameter determination at the Linear Collider}

In order to analyse the possible parameter reconstruction at the $\operatorname{ILC}_{500}$, we start with the NMSSM scenario of Table 1 and calculate the resulting cross sections for neutralino and chargino production for unpolarized beams and beam polarizations of $\left(\mathcal{P}_{e^{-}}, \mathcal{P}_{e^{+}}\right)=(-90 \%,+60 \%)$ and $(+90 \%,-60 \%)$ and assume $\Delta \mathcal{P}_{e^{-}} / \mathcal{P}_{e^{-}}=\Delta \mathcal{P}_{e^{+}} / \mathcal{P}_{e^{+}}=$ $0.5 \%$ [25]. At $\sqrt{s}=400 \mathrm{GeV}$ chargino pairs $\tilde{\chi}_{1}^{ \pm} \tilde{\chi}_{1}^{\mp}$ are produced, at $\sqrt{s}=500 \mathrm{GeV}$ the pairs $\tilde{\chi}_{1}^{ \pm} \tilde{\chi}_{1}^{\mp}$ and $\tilde{\chi}_{1}^{0} \tilde{\chi}_{2}^{0}$ are visible. Now the well-known strategies for determining the fundamental MSSM parameters in the gaugino/higgsino sector [6] are applied, taking into account experimental errors of $1.5 \%(2 \%)$ in the mass measurements of $\tilde{\chi}_{2,3}^{0}$, $\tilde{e}_{L, R}, \tilde{\nu}\left(\tilde{\chi}_{1}^{0}, \tilde{\chi}_{1}^{ \pm}\right)$and one standard deviation statistical errors for the cross sections [14] as given in Table 3. The statistical errors are based on a total luminosity of $500 \mathrm{fb}^{-1}$, which results in $\int \mathcal{L}=100 \mathrm{fb}^{-1}$ for each of the possible five polarization configurations $\left(\mathcal{P}_{e^{-}}, \mathcal{P}_{e^{+}}\right)=(0,0),(-90 \%,+60 \%),(-90 \%,-60 \%),(+90 \%,+60 \%)$ and $(+90 \%,-60 \%)$.

We determine the components of the chargino eigenstates $U_{11}$ and $V_{11}$ [26] from the measured polarized cross sections, which are bilinear quadratic functions of $U_{11}^{2}$ and $V_{11}^{2}$ and depend also on $m_{\tilde{\chi}_{1}^{ \pm}}$and $m_{\tilde{\nu}_{e}}$. The expressions for the chargino cross sections are analytically inverted [14]. In order to resolve ambiguities we assume that the process has been measured at two different energies, $\sqrt{s}=400$ and $500 \mathrm{GeV}$, and determine the components of the chargino eigenstates, which are consistent with the measured cross sections within the estimated error bounds of Table 3

$$
U_{11}^{2}=[0.84,1.0], \quad V_{11}^{2}=[0.83,1.0] .
$$




\begin{tabular}{|l|c|c|c|}
\hline$m_{\tilde{\chi}_{1}^{0}} / \mathrm{GeV}$ & $138 \pm 2.8$ & $m_{\tilde{e}_{L}} / \mathrm{GeV}$ & $240 \pm 3.6$ \\
$m_{\tilde{\chi}_{2}^{0}} / \mathrm{GeV}$ & $337 \pm 5.1$ & $m_{\tilde{e}_{R}} / \mathrm{GeV}$ & $220 \pm 3.3$ \\
$m_{\tilde{\chi}_{1}^{ \pm}} / \mathrm{GeV}$ & $139 \pm 2.8$ & $m_{\tilde{\nu}_{e}} / \mathrm{GeV}$ & $226 \pm 3.4$ \\
\hline \hline \multicolumn{2}{|c|}{$\sigma\left(e^{+} e^{-} \rightarrow \tilde{\chi}_{1}^{ \pm} \tilde{\chi}_{1}^{\mp}\right) / \mathrm{fb}$ at $\sqrt{s}=400 \mathrm{GeV}$} \\
\hline Unpolarized beams & $323.9 \pm 33.5$ \\
$\left(\mathcal{P}_{e^{-}}, \mathcal{P}_{e^{+}}\right)=(-90 \%,+60 \%)$ & $984.0 \pm 101.6$ \\
$\left(\mathcal{P}_{e^{-}}, \mathcal{P}_{e^{+}}\right)=(+90 \%,-60 \%)$ & $13.6 \pm 1.6$ \\
\hline \hline \multicolumn{2}{|c|}{$\sigma\left(e^{+} e^{-} \rightarrow \tilde{\chi}_{1}^{ \pm} \tilde{\chi}_{1}^{\mp}\right) / \mathrm{fb}$ at $\sqrt{s}=500 \mathrm{GeV}$} \\
\hline Unpolarized beams & $287.5 \pm 16.5$ \\
$\left(\mathcal{P}_{e^{-}}, \mathcal{P}_{e^{+}}\right)=(-90 \%,+60 \%)$ & $873.9 \pm 50.1$ \\
$\left(\mathcal{P}_{e^{-}}, \mathcal{P}_{e^{+}}\right)=(+90 \%,-60 \%)$ & $11.7 \pm 1.2$ \\
\hline \hline \multicolumn{2}{|c|}{$\sigma\left(e^{+} e^{-} \rightarrow \tilde{\chi}_{1}^{0} \tilde{\chi}_{2}^{0}\right) / \mathrm{fb}$ at $\sqrt{s}=500 \mathrm{GeV}$} \\
\hline Unpolarized beams & $4.0 \pm 1.2$ \\
$\left(\mathcal{P}_{e^{-}}, \mathcal{P}_{e^{+}}\right)=(-90 \%,+60 \%)$ & $12.1 \pm 3.8$ \\
$\left(\mathcal{P}_{e^{-}}, \mathcal{P}_{e^{+}}\right)=(+90 \%,-60 \%)$ & $0.2 \pm 0.1$ \\
\hline
\end{tabular}

Table 3: Masses with 1.5\% $\left(\tilde{\chi}_{2,3}^{0}, \tilde{e}_{L, R}, \tilde{\nu}\right)$ and $2 \%\left(\tilde{\chi}_{1}^{0}, \tilde{\chi}_{1}^{ \pm}\right)$uncertainty and cross sections with an error composed of the error due to the mass and polarization uncertainties and one standard deviation statistical error based on $\int \mathcal{L}=100 \mathrm{fb}^{-1}$, for both unpolarized beams and polarized beams with $\left(\mathcal{P}_{e^{-}}, \mathcal{P}_{e^{+}}\right)=(\mp 90 \%, \pm 60 \%)$ and $\Delta \mathcal{P}_{e^{ \pm}} / \mathcal{P}_{e^{ \pm}}=0.5 \%$, in analogy to the study in [14].

We then exploit additionally the polarized neutralino cross sections $\sigma\left(e^{+} e^{-} \rightarrow \tilde{\chi}_{1}^{0} \tilde{\chi}_{2}^{0}\right)$ at $\sqrt{s}=500 \mathrm{GeV}$ and the measured masses $m_{\tilde{\chi}_{1}^{0}}, m_{\tilde{\chi}_{2}^{0}}, m_{\tilde{e}_{L}}$ and $m_{\tilde{e}_{R}}$ in order to determine the parameters $M_{1}, M_{2}, \mu$ and $\tan \beta$ :

$$
\begin{aligned}
M_{1} & =377 \pm 42 \mathrm{GeV}, \\
M_{2} & =150 \pm 20 \mathrm{GeV}, \\
\mu & =450 \pm 100 \mathrm{GeV}, \\
\tan \beta & =[1,30] .
\end{aligned}
$$

Note that, in our mAMSB-inspired scenario with $M_{1}>M_{2}$, the crucial observable to determine the parameter $M_{1}$ is $m_{\tilde{\chi}_{2}^{0}}$, which can be clearly seen in Fig. 1127]. Since the heavier neutralino and chargino states are not produced, the parameters $\mu$ and $\tan \beta$ are determined with a considerable uncertainty.

In conclusion the measured masses and cross sections as experimental input from the ILC $_{500}$ are compatible with the MSSM. For the allowed ranges (4)-(7) of the MSSM parameters we now predict the heavier neutralino and chargino masses within the MSSM:

$$
m_{\tilde{\chi}_{3}^{0}}=443 \pm 107 \mathrm{GeV},
$$




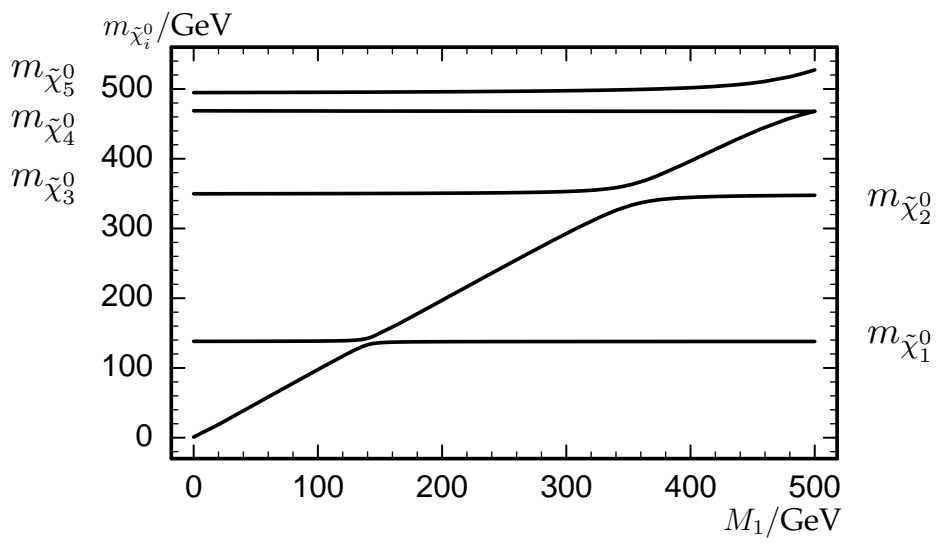

Figure 1: The neutralino masses $m_{\tilde{\chi}_{i}^{0}}, i=1, \ldots, 5$ as a function of $M_{1}$ in the NMSSM for the scenario of Table 1 .

$$
\begin{aligned}
m_{\tilde{\chi}_{4}^{0}} & =490 \pm 110 \mathrm{GeV}, \\
m_{\tilde{\chi}_{2}^{ \pm}} & =475 \pm 125 \mathrm{GeV} .
\end{aligned}
$$

We emphasize that although we started with an NMSSM scenario where $\tilde{\chi}_{2}^{0}$ and $\tilde{\chi}_{3}^{0}$ have large singlino admixtures (see Table 1), the strategy [5, 6] to determine the MSSM parameters is successful. All predictions for the heavier gaugino/higgsino masses are consistent in both models. In order to distinguish between the models, the measurement of the heavier neutralino and chargino masses at the LHC or with the $\mathrm{ILC}_{650}$ option is necessary. In our NMSSM scenario the gaugino admixture of $\tilde{\chi}_{3}^{0}$ probably allows its mass measurement at the LHC, whereas in the MSSM the neutralino $\tilde{\chi}_{3}^{0}$ always has a strong higgsino character within the predicted parameter ranges, eqs. (4)-(7), and would hardly be visible at the LHC.

\section{SUSY searches at the LHC}

The LHC has an excellent potential to discover supersymmetric particles. The expected large cross sections for squark and gluino production at the LHC give access to a large spectrum of coloured as well as non-coloured supersymmetric particles via the cascade decays. Simulations [28] exist e.g. for the mSUGRA point SPS1a [29]. It has also been worked out how to measure the masses of the heavier gauginos in cascade decays at the LHC [30]. Mass predictions from the ILC analysis lead to an increase of statistical sensitivity for the LHC analysis and open the possibility of identifying heavier gauginos even in marginal signals in the squark cascades [14]. However, since higgsino-like charginos and neutralinos do not couple to squarks, their detection via cascade decays is not possible. Direct pair production of charginos and neutralinos that occurs via Drell-Yan production is strongly suppressed for masses larger than about $300 \mathrm{GeV}$ as well as single neutralino production [31].

The mAMSB-inspired scenario of Table 1 contains nearly mass degenerate $\tilde{\chi}_{1}^{0}$ and $\tilde{\chi}_{1}^{ \pm}$with an LSP-NLSP mass difference in the difficult region for hadron colliders of 
$200 \mathrm{MeV}<m_{\tilde{\chi}_{1}^{ \pm}}-m_{\tilde{\chi}_{1}^{0}}<2 \mathrm{GeV}$ because the short-lived $\tilde{\chi}_{1}^{ \pm}$is not directly observable and its SM decay particles are softly emitted and suffer from a large background [18]. Applying specific cuts and using suitable kinematical variables, however, leads to a rather accurate measurement of the mass difference. A gain in accuracy can be obtained using the LSP and NLSP mass from ILC measurements. Since there exists no simulation for our chosen parameter point, we assume a precision for the heavier neutralino masses as in [30], motivated by supersymmetric searches in AMSB scenarios at the LHC [18]. A more detailed analysis will be performed in a forthcoming work [32].

The neutralinos $\tilde{\chi}_{2}^{0}$ and $\tilde{\chi}_{3}^{0}$ have a large bino-admixture and therefore appear in the squark decay cascades. The dominant decay mode of $\tilde{\chi}_{2}^{0}$ has a branching ratio $B R\left(\tilde{\chi}_{2}^{0} \rightarrow \tilde{\chi}_{1}^{ \pm} W^{\mp}\right) \sim 50 \%$, while for the $\tilde{\chi}_{3}^{0}$ decays $B R\left(\tilde{\chi}_{3}^{0} \rightarrow \tilde{\ell}_{L, R}^{ \pm} \ell^{\mp}\right) \sim 45 \%$ is largest. Since the heavier neutralinos, $\tilde{\chi}_{4}^{0}, \tilde{\chi}_{5}^{0}$, are mainly higgsino-like, no visible edges from these particles occur in the cascades. It is expected to see the edges for $\tilde{\chi}_{2}^{0} \rightarrow \tilde{\ell}_{R}^{ \pm} \ell^{\mp}$, $\tilde{\chi}_{2}^{0} \rightarrow \tilde{\ell}_{L}^{ \pm} \ell^{\mp}, \tilde{\chi}_{3}^{0} \rightarrow \tilde{\ell}_{R}^{ \pm} \ell^{\mp}$ and for $\tilde{\chi}_{3}^{0} \rightarrow \tilde{\ell}_{L}^{ \pm} \ell^{\mp}[32$, 33].

With a precise mass measurement of $\tilde{\chi}_{1}^{0}, \tilde{\chi}_{2}^{0}, \tilde{\ell}_{L, R}$ and $\tilde{\nu}$ from the $\operatorname{ILC}_{500}$ analysis, a clear identification and separation of the edges of the two gauginos at the LHC is possible without imposing specific model assumptions. We therefore assume a precision of about $2 \%$ for the measurement of $m_{\tilde{\chi}_{3}^{0}}$, in analogy to [30]:

$$
m_{\tilde{\chi}_{3}^{0}}=367 \pm 7 \mathrm{GeV} .
$$

Here the error assumption is rather conservative since contrary to the scenarios in [30] the branching ratio $B R\left(\tilde{q}_{R} \rightarrow \tilde{\chi}_{3}^{0} q\right) \times B R\left(\tilde{\chi}_{3}^{0} \rightarrow \tilde{\ell}_{L, R}^{ \pm} \ell^{\mp}\right)$ is very large [33].

The precise mass measurement of $\tilde{\chi}_{3}^{0}$ is compatible with the mass predictions of the $\mathrm{ILC}_{500}$. However, it is still to confirm that the measured particle is indeed the $\tilde{\chi}_{3}^{0}$ and not the $\tilde{\chi}_{4}^{0}$. The predicted $\tilde{\chi}_{3}^{0}$ in the MSSM is nearly a pure higgsino, which is typical for the constrained MSSM, and does not couple in the cascade decays, while the $\tilde{\chi}_{4}^{0}$ with a sufficiently large gaugino component could be measured in the cascades [14, 30]. In order to identify the particle and finally determine the masses and mixing characters of the heavy neutralinos one has to discuss the following cases:

- Assuming the measured particle to be $\tilde{\chi}_{3}^{0}$ and feeding it back in the ILC analysis lead to improved parameter determination and mass predictions for $m_{\tilde{\chi}_{4}^{0}}$ and $m_{\tilde{\chi}_{2}^{ \pm}}$. Using eq. (11) for the $\mathrm{ILC}_{500}$ analysis leads in our case, after rechecking with the allowed cross sections of $\tilde{\chi}_{1}^{0} \tilde{\chi}_{2}^{0}$ and $\tilde{\chi}_{1}^{+} \tilde{\chi}_{1}^{-}$production, to the precise mass predictions:

$$
m_{\tilde{\chi}_{4}^{0}}=[384,393] \mathrm{GeV} \text { and } m_{\tilde{\chi}_{2}^{ \pm}}=[360,380] \mathrm{GeV} \text {. }
$$

- Assuming the measured particle to be $\tilde{\chi}_{4}^{0}$ and feeding it back in the parameter determination of the ILC analysis lead to inconsistency with the measured cross sections of $\tilde{\chi}_{1}^{0} \tilde{\chi}_{2}^{0}$ and $\tilde{\chi}_{1}^{+} \tilde{\chi}_{1}^{-}$production.

The combined $\mathrm{LHC} / \mathrm{ILC}_{500}$ analysis therefore leads to a consistent interpretation of the measured particles in the cascades. However, a neutralino $\tilde{\chi}_{3}^{0}$ with sufficiently 
large gaugino admixture to couple to squarks is incompatible with the allowed parameter ranges of eqs. (4)-(7) in the MSSM. One should note that this result has been derived within the chargino/neutralino sector of the $\mathrm{CP}$-conserving unconstrained MSSM, without any assumption concerning the SUSY-breaking mechanism.

We point out that a measurement of the neutralino masses $m_{\tilde{\chi}_{1}^{0}}, m_{\tilde{\chi}_{2}^{0}}, m_{\tilde{\chi}_{3}^{0}}$ which can take place at the LHC alone is not sufficient to distinguish the SUSY models since rather similar mass spectra may exist. Both a model-independent analysis using cross section and masses at the ILC and the precisely measured masses from the cascade analysis at the LHC are needed for the identification of the particles. Furthermore, the results from the $\mathrm{LHC}$ and the $\mathrm{ILC}_{500}$ analyses and the precise predictions for the missing chargino/neutralino masses, eq. (12), motivates to apply immediately the lowluminosity but higher-energy option $\operatorname{ILC}_{650}^{\mathcal{L}=1 / 3}$, which finally could lead to the correct identification of the underlying model.

\section{The ILC $\mathrm{C}_{650}^{\mathcal{L}=1 / 3}$ option}

The ILC design provides the option to enhance instantaneously the energy at the cost of reduced luminosity. A centre-of-mass energy of $\sqrt{s}=650 \mathrm{GeV}$ at a third of the luminosity, i.e. $\int \mathcal{L}=33 \mathrm{fb}^{-1}$ per polarization configuration, does not require hardware changes in the experimental set-up but only running time.

The inconsistency of the MSSM analyses, the predicted higgsino-like $\tilde{\chi}_{3}^{0}$ and the measurement of this neutralino at the LHC, described in the previous sections may motivate this higher-energy option. The expected polarized and unpolarized cross sections, including the statistical error on the basis of one third of the luminosity of the $\mathrm{ILC}_{500}$, are given in Table 4. The neutralino $\tilde{\chi}_{3}^{0}$ as well as the higgsino-like heavy neutralino $\tilde{\chi}_{4}^{0}$ and the chargino $\tilde{\chi}_{2}^{ \pm}$are now accessible at the $\operatorname{ILC}_{650}^{\mathcal{L}=1 / 3}$. The production of $\tilde{\chi}_{1}^{0} \tilde{\chi}_{3}^{0}$ and $\tilde{\chi}_{1}^{0} \tilde{\chi}_{4}^{0}$ leads to promising rates at the $650 \mathrm{GeV}$ option while the heaviest neutralino $\tilde{\chi}_{5}^{0}$ is still too close to the threshold and the production cross sections are probably too small to be observed. The precise mass measurements of $\tilde{\chi}_{4}^{0}$ and $\tilde{\chi}_{2}^{ \pm}$lead to a clear identification of the supersymmetric model by comparing them with the predictions of eq. (12). The accuracy of $\mu_{\mathrm{eff}}=\lambda x$ is improved by the measurements of the cross sections for the associated $\tilde{\chi}_{1}^{ \pm} \tilde{\chi}_{2}^{\mp}$ production shown in Table 5 .

Finally the masses $m_{\tilde{\chi}_{1}^{0}}, m_{\tilde{\chi}_{2}^{0}}, m_{\tilde{\chi}_{3}^{0}}, m_{\tilde{\chi}_{4}^{0}}$ and $m_{\tilde{\chi}_{1}^{ \pm}}, m_{\tilde{\chi}_{2}^{ \pm}}$, as well as the cross sections, constitute the observables for a fit of the parameters $M_{1}, M_{2}, \tan \beta, \lambda$ and $\mu_{\text {eff }}=\lambda x, \kappa x$ of the gaugino/higgsino sector, which is, however, beyond the scope of this paper. Our analysis shows that the interplay between the two experiments is crucial for the determination of the supersymmetric model. It motivates the use of the low-luminosity, $\sqrt{s}=650 \mathrm{GeV}$, option of the ILC in order to resolve model ambiguities even at an early stage of the experiment and outlines future search strategies at an upgraded LHC as well as at the ILC at $1 \mathrm{TeV}$. 


\begin{tabular}{|c|c|c|c|}
\hline & \multicolumn{3}{|c|}{$\sigma\left(e^{+} e^{-} \rightarrow \tilde{\chi}_{1}^{0} \tilde{\chi}_{j}^{0}\right) / \mathrm{fb}$ at $\sqrt{s}=650 \mathrm{GeV}$} \\
\cline { 2 - 4 } & $j=3$ & $j=4$ & $j=5$ \\
\hline \hline Unpolarized beams & $12.2 \pm 0.6$ & $5.5 \pm 0.4$ & $\leq 0.02$ \\
\hline$\left(\mathcal{P}_{e^{-}}, \mathcal{P}_{e^{+}}\right)=(-90 \%,+60 \%)$ & $36.9 \pm 1.1$ & $14.8 \pm 0.7$ & $\leq 0.07$ \\
\hline$\left(\mathcal{P}_{e^{-}}, \mathcal{P}_{e^{+}}\right)=(+90 \%,-60 \%)$ & $0.6 \pm 0.1$ & $2.2 \pm 0.3$ & $\leq 0.01$ \\
\hline
\end{tabular}

Table 4: Expected cross sections for the associated production of the heavier neutralinos in the NMSSM scenario of Table 1 for the $\operatorname{ILC}_{650}^{\mathcal{L}=1 / 3}$ option with one sigma statistical error based on $\mathcal{L}=33 \mathrm{fb}^{-1}$ for both unpolarized and polarized beams.

\begin{tabular}{|c|c|}
\hline & $\sigma\left(e^{+} e^{-} \rightarrow \tilde{\chi}_{1}^{ \pm} \tilde{\chi}_{2}^{\mp}\right) / \mathrm{fb}$ at $\sqrt{s}=650 \mathrm{GeV}$ \\
\hline \hline Unpolarized beams & $2.4 \pm 0.3$ \\
\hline$\left(\mathcal{P}_{e^{-}}, \mathcal{P}_{e^{+}}\right)=(-90 \%,+60 \%)$ & $5.8 \pm 0.4$ \\
\hline$\left(\mathcal{P}_{e^{-}}, \mathcal{P}_{e^{+}}\right)=(+90 \%,-60 \%)$ & $1.6 \pm 0.2$ \\
\hline
\end{tabular}

Table 5: Expected cross sections for the associated production of the charginos in the NMSSM scenario of Table 1 for the $\operatorname{ILC}_{650}^{\mathcal{L}=1 / 3}$ option with one sigma statistical error based on $\mathcal{L}=33 \mathrm{fb}^{-1}$ for both unpolarized and polarized beams.

\section{Conclusion}

We have presented a scenario in the next-to-minimal supersymmetric standard model (NMSSM) that cannot be distinguished from the MSSM at the first stage of the International Linear Collider with $\sqrt{s}=500 \mathrm{GeV}$ by cross section and mass measurements. Although a light neutralino has a significant singlino component in the NMSSM, the masses of the accessible light neutralinos and charginos, as well as the production cross sections, are identical in the two models within experimental errors. The measurement of the masses of the heavier neutralinos and charginos in combined analyses of the experimental results at the LHC and at the higher-energy option $\operatorname{ILC}_{650}^{\mathcal{L}=1 / 3}$ with $\sqrt{s}=650 \mathrm{GeV}$ leads to a clear identification of the supersymmetric model.

\section{Acknowledgements}

We are very grateful to G. Polesello and P. Richardson for constructive discussions. We also thank J. Kalinowski for his critical reading of the manuscript. S.H. is supported by the Göran Gustafsson Foundation. This work is supported by the European Community's Human Potential Programme under contract HPRN-CT-2000-00149, by the 'Fonds zur Förderung der wissenschaftlichen Forschung' of Austria, FWF Project No. P16592-N02, and by the Deutsche Forschungsgemeinschaft (DFG) under contract No. FR 1064/5-2. 


\section{References}

[1] J.-E. Augustin et al., Executive Summary, International Technical Review Panel, August 2004, see also http: / / www . interactions.org/linearcollider/.

[2] J. G. Branson, D. Denegri, I. Hinchliffe, F. Gianotti, F. E. Paige and P. Sphicas [ATLAS and CMS Collaborations], Eur. Phys. J. direct C 4 (2002) N1.

[3] T. Abe et al. [American Linear Collider Working Group Collaboration], hep-ex/0106056:

TESLA Technical Design Report, Part III, eds. R.-D. Heuer, D. Miller, F. Richard and P. Zerwas, hep-ph/0106315;

K. Abe et al. [ACFA Linear Collider Working Group Collaboration], hep-ph/0109166.

[4] T. Tsukamoto, K. Fujii, H. Murayama, M. Yamaguchi and Y. Okada, Phys. Rev. D 51 (1995) 3153;

J. L. Feng, M. E. Peskin, H. Murayama and X. Tata, Phys. Rev. D 52 (1995) 1418 |hep-ph/9502260|.

[5] S. Y. Choi, A. Djouadi, M. Guchait, J. Kalinowski, H. S. Song and P. M. Zerwas, Eur. Phys. J. C 14 (2000) 535 |hep-ph/0002033|;

S. Y. Choi, A. Djouadi, H. K. Dreiner, J. Kalinowski and P. M. Zerwas, Eur. Phys. J. C 7 (1999) 123 [hep-ph/9806279].

[6] S. Y. Choi, J. Kalinowski, G. Moortgat-Pick and P. M. Zerwas, Eur. Phys. J. C 22 (2001) 563 |hep-ph/0108117]; Addendum, Eur. Phys. J. C 23 (2002) 769 |hep-ph/0202039|.

[7] H. P. Nilles, M. Srednicki and D. Wyler, Phys. Lett. B 120 (1983) 346;

J. P. Derendinger and C. A. Savoy, Nucl. Phys. B 237 (1984) 307;

M. Drees, Int. J. Mod. Phys. A 4 (1989) 3635;

J. R. Ellis, J. F. Gunion, H. E. Haber, L. Roszkowski and F. Zwirner, Phys. Rev. D 39 (1989) 844;

T. Elliott, S. F. King and P. L. White, Phys. Lett. B 314 (1993) 56 |hep-ph/9305282];

Phys. Rev. D 49 (1994) 2435 [hep-ph/9308309];

U. Ellwanger, M. Rausch de Traubenberg and C. A. Savoy, Phys. Lett. B 315 (1993) 331 [hep-ph/9307322];

P. N. Pandita, Phys. Lett. B 318 (1993) 338; Z. Phys. C 59 (1993) 575;

B. R. Kim, A. Stephan and S. K. Oh, Phys. Lett. B 336 (1994) 200.

[8] F. Franke and H. Fraas, Z. Phys. C 72 (1996) 309 [hep-ph/9511275]; Int. J. Mod. Phys. A 12 (1997) 479 [hep-ph/9512366];

F. Franke, H. Fraas and A. Bartl, Phys. Lett. B 336 (1994) 415 [hep-ph/9408217|.

[9] S. Y. Choi, D. J. Miller and P. M. Zerwas, Nucl. Phys. B 711 (2005) 83 |hep-ph/0407209]. 
[10] U. Ellwanger and C. Hugonie, Eur. Phys. J. C 5 (1998) 723 [hep-ph/9712300]; Eur. Phys. J. C 13 (2000) 681 [hep-ph/9812427].

[11] S. Hesselbach, F. Franke and H. Fraas, Phys. Lett. B 492 (2000) 140 |hep-ph/0007310|;

F. Franke and S. Hesselbach, Phys. Lett. B 526 (2002) 370 |hep-ph/0111285|; hep-ph/0210363.

[12] G. Moortgat-Pick, S. Hesselbach, F. Franke and H. Fraas, hep-ph/9909549.

S. Hesselbach, F. Franke and H. Fraas, hep-ph/0003272; Eur. Phys. J. C 23 (2002) 149 [hep-ph/0107080].

[13] W. Porod, Comput. Phys. Commun. 153 (2003) 275 [hep-ph/0301101];

R. Lafaye, T. Plehn and D. Zerwas, hep-ph/0404282, see also

http://sfitter.web.cern.ch/SFITTER/;

P. Bechtle, PhD Thesis, University of Hamburg, July 2004;

P. Bechtle, K. Desch and P. Wienemann, hep-ph/0412012, see also

http://www-flc.desy.de/fittino/.

[14] K. Desch, J. Kalinowski, G. Moortgat-Pick, M. M. Nojiri and G. Polesello, JHEP 0402 (2004) 035 |hep-ph/0312069|;

B. C. Allanach et al. [Beyond the Standard Model Working Group Collaboration], hep-ph/0402295:

G. Moortgat-Pick, hep-ph/0406180

[15] U. Ellwanger, J. F. Gunion and C. Hugonie, JHEP 0502 (2005) 066 |hep-ph/0406215|.

[16] P. Skands et al., JHEP 0407 (2004) 036 [hep-ph/0311123].

[17] L. Randall and R. Sundrum, Nucl. Phys. B 557 (1999) 79 [hep-th/9810155];

G. F. Giudice, M. A. Luty, H. Murayama and R. Rattazzi, JHEP 9812 (1998) 027 |hep-ph/9810442];

A. Pomarol and R. Rattazzi, JHEP 9905 (1999) 013 |hep-ph/9903448|.

[18] A. J. Barr, C. G. Lester, M. A. Parker, B. C. Allanach and P. Richardson, JHEP 0303 (2003) 045 [hep-ph/0208214|.

[19] C. Hensel, DESY-THESIS-2002-047.

[20] J. F. Gunion and S. Mrenna, Phys. Rev. D 64 (2001) 075002 [hep-ph/0103167|;

[21] G. Belanger, F. Boudjema, A. Pukhov and A. Semenov, hep-ph/0405253

[22] U. Ellwanger and C. Hugonie, Eur. Phys. J. C 25 (2002) 297 |hep-ph/9909260|;

D. J. Miller, R. Nevzorov and P. M. Zerwas, Nucl. Phys. B 681 (2004) 3 |hep-ph/0304049.

[23] D. J. Miller and S. Moretti, hep-ph/0403137 
[24] U. Ellwanger, J. F. Gunion, C. Hugonie and S. Moretti, hep-ph/0305109:

J. F. Gunion and M. Szleper, hep-ph/0409208.

[25] For details see the Polarization Working Group report of the 'POWER group', http://www.ippp.dur.ac.uk/ ^gudrid/power.

[26] H. E. Haber and G. L. Kane, Phys. Rept. 117 (1985) 75.

[27] G. Moortgat-Pick, A. Bartl, H. Fraas and W. Majerotto, hep-ph/0002253.

[28] The ATLAS Collaboration, ATLAS TDR 15, CERN/LHCC/99-15 (1999);

M. Chiorboli and A. Tricomi, CMS-NOTE-2004-029;

B. K. Gjelsten, D. J. Miller and P. Osland, JHEP 0412 (2004) 003 |hep-ph/0410303;

G. Weiglein et al. [LHC/LC Study Group], hep-ph/0410364.

[29] B. C. Allanach et al., Eur. Phys. J. C 25 (2002) 113 [hep-ph/0202233];

N. Ghodbane and H. U. Martyn, hep-ph/0201233:

see also http: //www.cpt.dur.ac.uk/〜georg/sps/.

[30] G. Polesello, J. Phys. G 30 (2004) 1185.

[31] G. J. Gounaris, J. Layssac, P. I. Porfyriadis and F. M. Renard, Phys. Rev. D 71 (2005) 075012 [hep-ph/0411366].

[32] H. Fraas, F. Franke, S. Hesselbach, G. Moortgat-Pick and G. Polesello, in preparation.

[33] G. Polesello, private communication. 